\title{
Prognostic value of the 2018 FIGO staging system for cervical cancer patients with surgical risk factors
}

Ding-Ding Yan

Qiu Tang

Jian-Hong Chen

Ye-Qiang Tu

Xiao-Juan Lv

Department of Gynecologic Oncology, Zhejiang Cancer Hospital, Hangzhou, People's Republic of China
Correspondence: Qiu Tang

Department of Gynecologic Oncology,

Zhejiang Cancer Hospital, No. 38, Guangji

Road, Bansan Bridge, Gongshu District,

Hangzhou 310022, People's Republic of China

$\mathrm{Tel}+8657188128218$

Fax +865 718 812 2508

Email tangqiu@zjcc.org.cn
This article was published in the following Dove Press journal: Cancer Management and Research

Objective: To determine the predictive value of the 2018 International Federation of Gynecology and Obstetrics (FIGO) staging system for cervical cancer patients with surgical risk factors.

Methods: Data of 662 cervical cancer patients (stages IB and IIA) with surgical risk factors treated at Zhejiang Cancer Hospital between 2008 and 2011 were retrospectively reviewed. Univariate log-rank test and multivariate Cox regression models were adopted to evaluate the relationship between 2018 FIGO stage and survival.

Results: On re-staging of patients, $17.3 \%, 44.5 \%, 25.4 \%$, and $37.1 \%$ of the patients with FIGO 2009 stage IB1, IB2, IIA1, and IIA2, respectively, were upgraded to FIGO 2018 IIIC1P stage, and $2.1 \%, 3.0 \%, 3.1 \%$, and $2.1 \%$ patients, respectively, were upgraded to IIIC2P stage. The 5-year overall survival (OS) rates of patients with FIGO 2018 stage IB1, IB2, IB3, IIA1, IIA2, IIIC1P, and IIIC2P were 95.3\%, 95.1\%, 90.4\%, 92.4\%, 86.4\%, 81.9\%, and $56.3 \%$, respectively. The 5-year progression-free survival (PFS) rates were $94.0 \%$, $91.0 \%, 88.5 \%, 91.4 \%, 86.4 \%, 79.5 \%$, and $43.8 \%$, respectively. The 5-year OS rates of patients with 1-2 positive pelvic lymph nodes (PLNs) and those with $>2$ positive PLNs were $86.0 \%$ and $73.7 \%$, respectively, and the 5-year PFS rates were $84.2 \%$ and $70.2 \%$, respectively. OS and PFS of patients with 1-2 positive PLNs in stage IIIC1P were similar to those of patients in stage IIA2 without lymph node metastasis, but significantly better than those of patients with $>2$ positive PLNs. Multivariate analysis showed FIGO 2018 stage to be an independent prognostic factor for OS and PFS.

Conclusion: The 2018 FIGO staging system for cervical cancer appears to be useful for predicting prognosis of patients with risk factors after radical surgery. Survival of stage IIA1 patients is better than that of stage IB3 patients. Stage IIIC1 is not homogenous; survival in stage IIIC1P depends on the number of positive PLNs.

Keywords: cervical cancer, 5-year overall survival, 5-year progression-free survival, FIGO staging system

\section{Background}

With widespread implementation of cervical cancer screening programs worldwide, there has been an increase in the number of early cervical cancers being detected. For patients with stage IB-IIA cervical cancer, radical hysterectomy plus pelvic lymphadenectomy is considered the treatment of choice. The oncologist determines the adjuvant treatment after operation according to the risk factors of patients. ${ }^{1,2,3,4,5}$ Although surgical stage is a prognostic factor for cervical cancer patients, the International Federation of Gynecology and Obstetrics (FIGO) Committee has been using only 
clinical criteria for staging of cervical cancer because this was considered more practicable in low-resource countries. Surgical risk factors were not taken into account for clinical staging and therefore, until recently, the FIGO clinical stage had little prognostic value in IB-IIA cervical cancer patients. ${ }^{6}$ However, after considering the improvements in medical technology worldwide, the FIGO Committee on Gynecologic Oncology decided to include surgical risk factors and lymph node status in the 2018 FIGO staging system. ${ }^{7}$ Few studies have attempted to validate the FIGO 2018 staging system. ${ }^{8}$ The purpose of this retrospective study was to determine the prognostic value of the 2018 FIGO staging system in cervical cancer patients with surgical risk factors who were staged as IB-IIA disease by the FIGO 2009 criteria.

\section{Patients and methods}

\section{Ethics statement}

This study was approved by the institutional ethical review board of the Zhejiang Cancer Hospital, Zhejiang, China. All procedures were performed in concordance with the tenets of the Helsinki declaration. Written informed consent was obtained from all patients.

\section{Patients}

Clinical data of 662 patients with FIGO 2009 stage IB-IIA cervical cancer treated in Zhejiang Cancer Hospital between 2008 and 2011 were retrospectively reviewed. All of these patients were receiving treatment for the first time. Table 1 shows the patient and tumor characteristics. Table 2 shows the distribution of risk factors in patients with different FIGO 2018 stages.

\section{Staging}

All patients were preoperatively staged by the FIGO 2009 criteria, which is based on clinical and imaging criteria and include the findings on 1) gynecological examination by radiotherapists with specialization in gynecologic tumors and 2) chest radiography $\mathrm{CT}$, abdomen $\mathrm{CT}$, and pelvis $\mathrm{CT}$ or MRI to determine the extent of primary tumor and to exclude distant metastasis. For this study, we re-staged all patients by the FIGO 2018 staging system.

\section{Surgery}

All 662 patients underwent type III hysterectomy with pelvic lymphadenectomy. Para-aortic lymph node (PALN) resection was not routinely performed as it can increase operation time and complications; it was performed only if intraoperative
Table I Patient and tumor characteristics $(n=662)$

\begin{tabular}{|c|c|}
\hline Characteristics & \\
\hline Median age, years, (range) & $45(2 I-74)$ \\
\hline \multicolumn{2}{|l|}{ Pathologic type, n (\%) } \\
\hline Squamous cell & 555 (83.9\%) \\
\hline Adenocarcinoma & 77 (1 I.6\%) \\
\hline Adenosquamous & 30 (4.5\%) \\
\hline \multicolumn{2}{|l|}{ Tumor size, n (\%) } \\
\hline$\leq 4.0 \mathrm{~cm}$ & 472 (7I.3\%) \\
\hline$>4 \mathrm{~cm}$ & 190 (28.7\%) \\
\hline \multicolumn{2}{|l|}{ DSI, n (\%) } \\
\hline$\leq \mathrm{I} / 2$ & 309 (46.7\%) \\
\hline$>1 / 2$ & $353(53.3 \%)$ \\
\hline \multicolumn{2}{|l|}{ LVSI, n (\%) } \\
\hline Negative & $374(56.5 \%)$ \\
\hline Positive & $288(43.5 \%)$ \\
\hline \multicolumn{2}{|l|}{ SM status, n (\%) } \\
\hline Negative & $646(97.6 \%)$ \\
\hline Positive & $16(2.4 \%)$ \\
\hline \multicolumn{2}{|l|}{ PI, n (\%) } \\
\hline Negative & $646(97.6 \%)$ \\
\hline Positive & $16(2.4 \%)$ \\
\hline \multicolumn{2}{|l|}{ Nodal metastasis, n (\%) } \\
\hline Negative & 475 (7I.8\%) \\
\hline Positive & $187(28.2 \%)$ \\
\hline \multicolumn{2}{|l|}{ Adjuvant therapy, $\mathrm{n}(\%)$} \\
\hline RT & I8I (27.3\%) \\
\hline CCRT & $283(42.7 \%)$ \\
\hline No & $198(30.0 \%)$ \\
\hline \multicolumn{2}{|l|}{ FIGO 2009 stage, n (\%) } \\
\hline$|B|$ & $336(50.8 \%)$ \\
\hline IB2 & 99 (I5.0\%) \\
\hline$|I A|$ & $130(19.6 \%)$ \\
\hline IIA2 & 97 (14.6\%) \\
\hline \multicolumn{2}{|l|}{ FIGO 2018 stage, n (\%) } \\
\hline$|B|$ & 149 (22.5\%) \\
\hline IB2 & $122(18.4 \%)$ \\
\hline IB3 & $91(13.8 \%)$ \\
\hline$\| A \mid$ & $54(8.2 \%)$ \\
\hline IIA2 & 59 (8.9\%) \\
\hline IIICIP & I7I (25.8\%) \\
\hline $\mathrm{IIIC} 2 \mathrm{P}$ & $16(2.4 \%)$ \\
\hline
\end{tabular}

Abbreviations: DSI, Depth of stromal invasion; LVSI, lymphovascular space invasion; SM, surgical margin; PI, parametrial invasion; RT, radiotherapy; CCRT, concurrent chemoradiotherapy; FIGO, International Federation of Gynecology and Obstetrics.

frozen section examination showed positive common iliac LN or if intraoperative palpation suggested PALN involvement. 
Table 2 Risk factors in different FIGO 2018 stages

\begin{tabular}{|c|c|c|c|c|c|c|}
\hline \multirow[b]{2}{*}{ FIGO20I8 stage } & \multicolumn{6}{|c|}{ Risk factor, n (\%) } \\
\hline & Large tumor & DSI & LVSI & LNM & PI & Positive SM \\
\hline$|\mathrm{B}|$ & $0(0)$ & $13(8.7)$ & $22(14.8)$ & 0 & 0 & I (0.7) \\
\hline IB2 & $0(0)$ & $55(45.1)$ & $50(4 \mid .0)$ & 0 & 0 & $2(1.6)$ \\
\hline IB3 & $52(100.0)$ & $35(67.3)$ & $19(36.5)$ & 0 & $2(3.8)$ & $3(5.8)$ \\
\hline IIAI & 0 & $4 I(44 . I)$ & $23(24.7)$ & 0 & $\mathrm{I}(\mathrm{I} . \mathrm{I})$ & $5(5.4)$ \\
\hline IIA2 & $59(100.0)$ & $50(84.8)$ & $26(44.1)$ & 0 & $2(3.4)$ & $3(5.1)$ \\
\hline IIICIP & $74(43.3)$ & $144(84.2)$ & I $33(77.8)$ & $|7|(100.0)$ & $9(5.3)$ & $2(1.2)$ \\
\hline $\mathrm{IIIC2P}$ & $5(2 \mid .3)$ & $15(93.8)$ & $15(93.8)$ & $16(100.0)$ & $2(12.5)$ & $0(0)$ \\
\hline
\end{tabular}

Abbreviations: FIGO, International Federation of Gynecology and Obstetrics; DSI, depth of stromal invasion; LVSI, lymphovascular space invasion; LNM, lymph node metastasis; PI, parametrial invasion; SM, surgical margin.

\section{Radiotherapy}

Postoperative total pelvic radiotherapy was administered for the patients with at least one of the following risk factors: positive surgical margin, parametrial invasion (PI), lymph node metastasis, lymphovascular space invasion (LVSI), depth of stromal invasion (DSI) $>1 / 2$, and tumor size $>4 \mathrm{~cm}$. Extended-field radiotherapy was administered only when common iliac LN or PALN metastasis was confirmed; the entire pelvis and para-aortic extendedfield were irradiated with 4,500-5,040 cGy in 25-28 fractions. Parametrial boost was administered for 5 patients with positive parametrial margins. Intracavitary radiotherapy with high-dose-rate Ir-192 was given to 11 patients with positive vaginal margins. Brachytherapy dose was $20-25$ Gy in 4-5 fractions.

\section{Chemotherapy}

None of the patients received neoadjuvant chemotherapy. Concurrent chemotherapy was administered to 283 patients; these included 113 patients who received 4-5 cycles of single cisplatin chemotherapy at a dose $40 \mathrm{mg} /$ $\mathrm{m}^{2}$ weekly; 137 patients who received 2 cycles of fluorouracil plus cisplatin (consisting of $1,000 \mathrm{mg} / \mathrm{m}^{2}$ of fluorouracil, continuous intravenous infusion for 1-4 days plus $60-65 \mathrm{mg} / \mathrm{m}^{2}$ of cisplatin, intravenous infusion on day 1); and 33 patients who received 2 cycles of chemotherapy with paclitaxel plus cisplatin regimen (consisting of $135 \mathrm{mg} / \mathrm{m}^{2}$ paclitaxel, intravenous infusion on day 1 , plus $60 \mathrm{mg} / \mathrm{m}^{2}$ cisplatin, intravenous infusion on day 1).

\section{Follow-up}

Patients were regularly reviewed as outpatients. Diagnosis of recurrence was based on CT or MRI findings; as far as possible the diagnosis was pathologically confirmed. Recurrence included local pelvic or vaginal recurrence and distant metastasis.

\section{Statistical methods}

SPSS 19.0 (IBM Corp., Armonk, NY, USA) was used for data analysis. The Kaplan-Meier method was used to calculate overall survival (OS) and progression-free survival (PFS). The log-rank test was used to compare the differences in survival. Cox proportional hazard regression model was used for multivariate analysis to identify the independent prognostic factors for survival. Statistical significance was at $p \leq 0.05$.

\section{Results}

\section{Follow-up and survival outcomes}

Last follow-up was in February 2017. Median follow-up was for 68 months (range, 4-96 months). The overall follow-up rate was $94.4 \%$; 37 patients were lost to followup. There were 76 deaths during the follow-up period. Recurrence was diagnosed in 89 patients: 45 had local recurrence, 42 had distant metastasis, and 2 had simultaneous local recurrence and distant metastasis.

\section{Change of FIGO stage}

Table 3 shows the distribution of stages by FIGO 2009 and FIGO 2018 criteria. Preoperative staging of the 662 patients by the FIGO 2009 criteria showed stage IB1 in 336 patients, stage IB2 in 99 patients, stage IIA1 in 130 patients, and stage IIA2 in 97 patients. Re-staging by the FIGO 2018 showed stage IB1 in 149 patients, stage IB2 in 122 patients, stage IB3 in 91 patients, stage IIA1 in 54 patients, stage IIA2 in 59 patients, stage IIIC1P in 171 patients, and stage IIIC2P in 16 patients. Of 336 
Table 3 Distribution of stages by FIGO 2009 and FIGO 2018 criteria

\begin{tabular}{|c|c|c|c|c|c|c|c|}
\hline \multirow[t]{2}{*}{ FIGO 2009 stage } & \multicolumn{7}{|c|}{ FIGO 2018 stage } \\
\hline & |B | & IB2 & IB3 & IIAI & IIA2 & IIICIP & IIIC2P \\
\hline$|\mathrm{B}|$ & I 49 (44.3\%) & $122(36.3 \%)$ & 0 & 0 & 0 & $58(17.3 \%)$ & 7 (2.1\%) \\
\hline IB2 & & & $52(52.5 \%)$ & 0 & 0 & 44 (44.5\%) & $3(3.0 \%)$ \\
\hline$\| A \mid$ & 0 & 0 & 0 & 93 (71.5\%) & 0 & 33 (25.4\%) & $4(3.1 \%)$ \\
\hline IIA2 & 0 & 0 & 0 & 0 & 59 (60.8\%) & $36(37.1 \%)$ & $2(2.1 \%)$ \\
\hline
\end{tabular}

Abbreviation: FIGO, International Federation of Gynecology and Obstetrics.

patients with FIGO 2009 stage IB1, 58 (17.3\%) had PLN metastasis and 7 (2.1\%) had PALN metastasis. Of the 99 patients with FIGO 2009 stage IB2, 44 (44.5\%) had PLN metastasis and $3(3.0 \%)$ had PALN metastasis. Of the 130 patients with FIGO 2009 stage IIA1, 33 (25.4\%) had PLN metastasis and 4 (3.1\%) had PALN metastasis. Of the 97 patients with FIGO 2009 stage IIA2, 36 (37.1\%) had PLN metastasis and 2 (2.1\%) had PALN metastasis.

\section{Survival analysis by FIGO 2018 stage}

The 5-year OS rates of patients with FIGO 2018 stage IB1, IB2, IB3, IIA1, IIA2, IIIC1P, and IIIC2P were 95.3\%, 95.1\%, $90.4 \%, 92.4 \%, 86.4 \%, 81.9 \%$, and $56.3 \%$, respectively. The 5-year PFS rates of patients with these stages were 94.0\%, $91.0 \%, 88.5 \%, 91.4 \%, 86.4 \%, 79.5 \%$, and $43.8 \%$, respectively. Survival declined with increase in FIGO 2018 stage, but the survival of stage IIA1 patients was better than that of stage IB3 patients. Survival rates were lower for patients with positive lymph nodes than for patients with negative lymph nodes; the difference between patients with negative lymph nodes and patients with PALN metastasis was statistically significant $(p<0.001)$. Subgroup analysis was performed to determine whether the number of PLN metastases affected survival in patients within IIIC1 stage. The 5-year OS and PFS were significantly higher in patients with 1-2 positive PLNs than in patients with $>2$ positive PLNs (OS: $86.0 \%$ vs $73.7 \%, p=0.047$; and PFS: $84.2 \%$ vs $70.2 \%, p=0.016$ ); however, survival rates were comparable between stage IIIC1P with 1-2 positive PLNs and patients with stage IIA2. Figure 1 shows the survival curves for the different FIGO 2018 stages and for stage IIIC1 patients with 1-2 and $>2$ positive PLNs.

\section{FIGO 2018 stage and prognosis}

Univariate analysis of seven potential prognostic factors was performed. DSI, LVSI, surgical margin, and FIGO 2018 stage were significantly associated with OS. Tumor size, DSI, LVSI, surgical margin, and FIGO 2018 stage were significantly associated with PFS. Table 4 shows the results of univariate analysis.

Cox proportional hazard analysis showed that surgical margin (hazard ratio $[\mathrm{HR}]=3.249,95 \% \mathrm{CI}: 1.279-8.249$; $p=0.013$ ) and FIGO 2018 stage (IIIC1P vs IB1: $\mathrm{HR}=2.597$, 95\% CI: $1.055-6.393 ; p=0.038$; IIIC2P vs IB1: HR=9.773, 95\% CI: 3.340-25.598; $p<0.001)$ were independent prognostic factors for OS. Surgical margin (hazard ratio [HR] $=3.697,95 \%$ CI: $0.711-2.148 ; p=0.007$ ) and FIGO 2018 stage (IIIC1P vs IB1: HR=3.246, 95\% CI: $1.202-8.763$; $p=0.020$; IIIC2P vs IB1: $\mathrm{HR}=11.588,95 \% \mathrm{CI}: 3.594-$ $37.358 ; p<0.001)$ were independent prognostic factors for PFS.

\section{Discussion}

The FIGO criteria have been used since 1958 for staging of cervical cancer. ${ }^{9}$ Until recently, only clinical and imaging findings were used for staging. The 2018 FIGO criteria, for the first time, included surgical risk factors in the staging system. ${ }^{7}$ This change mainly affects the staging of early cervical cancer. In this study, we examined how the change affects the prognostic power of the FIGO staging system.

When the patients in our study were re-staged by the new system, $17.3 \%, 44.5 \%, 25.4 \%$, and $37.1 \%$ of the patients with FIGO 2009 stages IB1, IB2, IIA1, and IIA2 were upgraded to FIGO 2018 IIIC1P stage, and $2.1 \%$, $3.0 \%, 3.1 \%$, and $2.1 \%$ of the patients with the abovementioned FIGO 2009 stages were upgraded to FIGO 2018 IIIC2P stage, respectively. Previous studies have found that the lymph node metastasis rate increases with increase in FIGO clinical stage. ${ }^{10-12}$ However, we noted that the lymph node metastasis rate in stage IB2 was higher than that in stage IIA. Matsuo et al have reported similar results. $^{13}$

Previous studies have shown that while, generally, survival declines significantly with increase in FIGO 
A

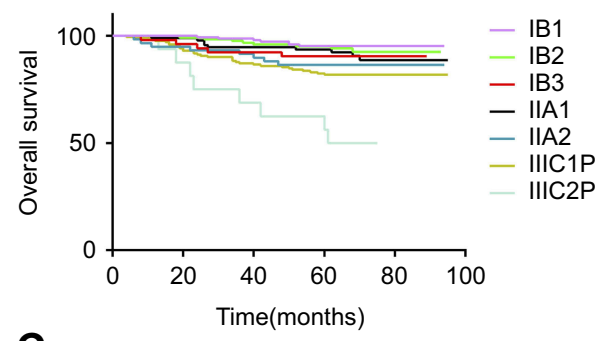

C

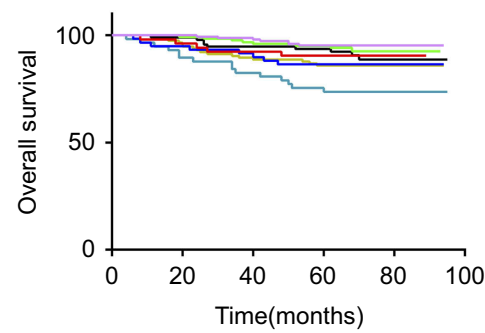

B

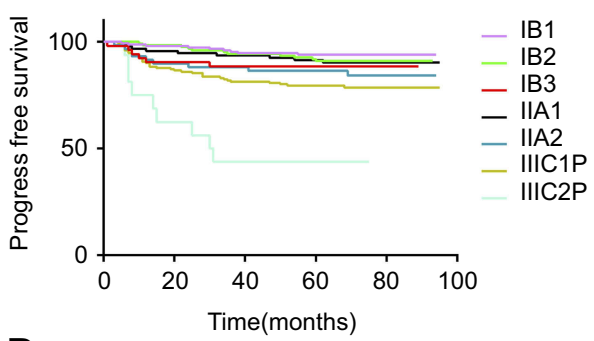

D

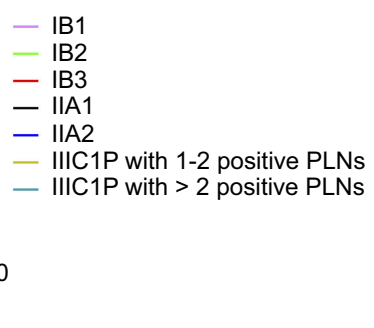

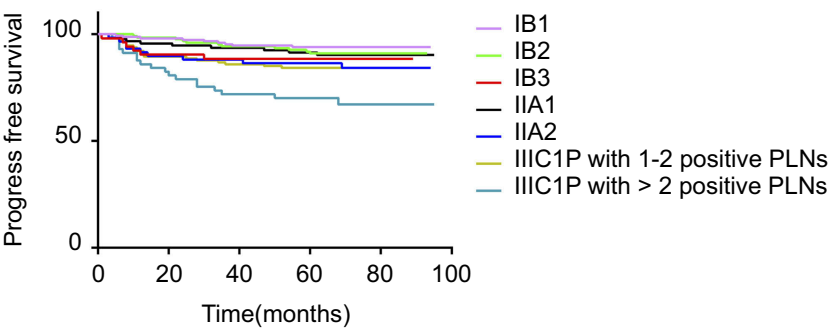

Figure I (A and B) PFS and OS curves for 2018 FIGO stages. (C and D) PFS and OS curves for patients with different numbers of metastatic PLNs.

Abbreviations: OS, overall survival; PFS, progress-free survival; RT, radiotherapy; FIGO, International Federation of Gynecology and Obstetrics; PLN, pelvic lymph node.

stage, survival did not vary significantly among patients in stages IB-IIA. ${ }^{6}$ This is because the previous FIGO staging system does not take into account the influence of clinicopathological factors. PLN metastasis is closely related to recurrence and prognosis of cervical cancer. In previous studies, the 5-year OS was $95 \%$ in stage IB-IIA cervical cancer patients without PLN metastasis vs $78 \%$ in stage IB-IIA patients with PLN metastasis. ${ }^{14,15}$ PALN metastasis has greater impact on survival than PLN metastasis. The Radiation Therapy Oncology Group (RTOG) 92-10 study showed that 2-year disease-free survival rate declined from $64 \%$ in patients without PALN metastasis to $14 \%$ in patients with PALN metastasis. ${ }^{16}$ In another multicenter clinical study, ${ }^{17}$ the 3-year OS was $89 \%$ in stage IB2-IVA patients without PALN metastasis vs $40 \%$ in IB2-IVA patients with PALN metastasis. The FIGO Committee added IIIC1P and IIIC2P to FIGO 2018 staging criteria considering the

Table 4 Results of univariate analysis

\begin{tabular}{|c|c|c|c|c|c|c|}
\hline \multirow[t]{2}{*}{ Variable } & \multicolumn{3}{|l|}{ OS } & \multicolumn{3}{|l|}{ PFS } \\
\hline & HR & $95 \% \mathrm{Cl}$ & p value & HR & $95 \% \mathrm{Cl}$ & $p$ value \\
\hline Tumor size $(>4 \mathrm{~cm}$ vs. $\leq 4.0 \mathrm{~cm}$ ) & 1.535 & $0.959-2.455$ & 0.074 & 1.586 & I.029-2.446 & 0.037 \\
\hline DSI $(>\mathrm{I} / 2$ vs. $\leq \mathrm{I} / 2)$ & 2.269 & I.382-3.725 & 0.001 & 2.397 & $1.510-3.806$ & $<0.001$ \\
\hline LVSI (positive vs. negative) & 2.106 & $1.329-3.336$ & 0.002 & 2.143 & $1.400-3.279$ & $<0.001$ \\
\hline SM (positive vs. negative) & 3.181 & $1.283-7.884$ & 0.012 & 2.714 & $1.101-6.695$ & 0.030 \\
\hline PI (positive vs. negative) & 0.532 & $0.074-3.824$ & 0.530 & 0.939 & $0.231-3.815$ & 0.930 \\
\hline $\begin{array}{l}\text { Histologic type (adenocarcinoma and } \\
\text { adenosquamous carcinoma vs. SCC) } \\
2018 \text { FIGO stage }\end{array}$ & 1.074 & $0.591-1.952$ & 0.815 & 1.139 & $0.663-1.958$ & 0.636 \\
\hline $\mid \mathrm{B} 2$ vs. IBI & 1.402 & $0.508-3.867$ & 0.514 & 1.494 & $0.619-3.606$ & 0.372 \\
\hline $\mid \mathrm{B} 3$ vs. IBI & 2.103 & $0.667-6.627$ & 0.204 & 2.003 & $0.713-5.629$ & 0.187 \\
\hline$\| A \mid$ vs. IBI & 2.079 & $0.774-5.583$ & 0.146 & 1.621 & $0.644-4.085$ & 0.305 \\
\hline IIA2 vs. IBI & 3.022 & $1.095-8.334$ & 0.033 & 2.656 & I.054-6.692 & 0.038 \\
\hline IIICIP vs. IBI & 4.170 & $|.836-9.47|$ & 0.001 & 3.811 & $1.836-7.912$ & $<0.001$ \\
\hline IIIC2P vs. IBI & 13.902 & $5.037-38.368$ & $<0.001$ & 13.440 & $5.325-33.920$ & $<0.001$ \\
\hline
\end{tabular}

Abbreviations: OS, Overall survival; PFS, progression-free survival; DSI, depth of stromal invasion; LVSI, lymphovascular space invasion; SM, surgical margin; PI, parametrial invasion; SCC, squamous cell carcinoma; FIGO, International Federation of Gynecology and Obstetrics. 
effect of lymph node metastasis on prognosis. $^{7}$ In our study, after re-staging of patients by the FIGO 2018 system, survival showed significant decline with increase in stage, which of those with IIIC1P and IIIC2P decreased more significantly. The 5-year OS and PFS rates were $81.9 \%$ and $79.5 \%$, respectively, in IIIC1P stage vs $56.3 \%$ and $43.8 \%$, respectively, in IIIC2P stage. This is consistent with previous studies. ${ }^{14-17}$ Thus, the FIGO 2018 staging criteria can reflect the effect of lymph node metastasis on the survival of patients and improve prognosis prediction.

Previous studies have reported that large tumor size, DSI, LVSI, lymph node metastasis, PI, and positive surgical margin are prognostic factors in early cervical cancer, ${ }^{1-5}$ and that the FIGO clinical stage has no prognostic significance. ${ }^{6}$ In the present study, we did not include lymph node metastasis as a variable in multivariate analysis because the FIGO 2018 staging criteria include lymph node metastasis. Multivariate analysis showed FIGO 2018 stage to be an independent prognostic factor for OS and PFS. Thus, our study demonstrates the prognostic value of surgical staging in early cervical cancer.

In the present study, FIGO 2018 stage was shown to be a significant predictor of survival. Survival in stage IB (IB1, IB2, and IB3) decreased gradually with increase in stage, especially that of stage IB3. Matsuo et al reported that the FIGO 2018 stage is closely related to cause-specific survival in stage IB disease. ${ }^{8}$ Our results support their finding. However, we found that the survival of patients with stage
IIA1 was better than that of stage IB3. We analyzed the reasons for this. As Table 5 shows, the number of risk factors that affected the survival was significantly higher in stage IB3 patients than in stage IIA1 patients. In this study determination of IB-IIA stage was based only on the size of the tumor and the status of vaginal involvement; risk factors such as LVSI, DSI, PI, and positive resection margin were not considered. Thus, it appears that FIGO 2018 stages IB and IIA, which do not take into account postoperative risk factors, may not correctly predict survival and prognosis.

In previous studies, the number of positive PLNs has been shown to affect prognosis. Inoue et $\mathrm{al}^{18}$ found the 5 -year OS of patients with no PLN involvement, 1 positive PLN, 2-3 positive PLN, and $>4$ positive PLN to be $89 \%$, $81 \%, 41 \%$, and $23 \%$, respectively. Sakuragi et al ${ }^{19}$ reported that the 5-year OS rates of patients with one and multiple lymph node metastases were $84.9 \%$ and $26.5 \%$, respectively; they found no difference in survival between patients with one lymph node metastasis and those with no lymph node metastasis. Takeda et $\mathrm{al}^{20}$ reported that patients with $>2$ positive PLNs had relatively poor prognosis, with 5 -year OS being $20.2 \%$ in those with stage IB-IIB disease. Our findings were similar; we found that OS and PFS rates of patients with 1-2 positive PLNs in stage IIIC1P were similar to those of patients in stage IIA2 without lymph node metastasis, but significantly higher than those of patients with $>2$ positive PLNs. This clearly shows that stage IIIC1P cervical cancer is not homogenous; survival in stage IIIC1P varies with the

Table 5 Results of multivariate analysis

\begin{tabular}{|c|c|c|c|c|c|c|}
\hline \multirow[t]{2}{*}{ Variable } & \multicolumn{3}{|l|}{ OS } & \multicolumn{3}{|l|}{ PFS } \\
\hline & HR & $95 \% \mathrm{Cl}$ & p value & HR & $95 \% \mathrm{Cl}$ & p value \\
\hline Tumor size ( $\geq 4 \mathrm{~cm}$ vs. $<4.0 \mathrm{~cm}$ ) & 1.047 & $0.580-1.890$ & 0.879 & 0.950 & $0.504-1.79 \mid$ & 0.874 \\
\hline DSI (>I/2 vs. $\leq \mathrm{I} / 2)$ & 1.398 & $0.794-2.462$ & 0.246 & 1.262 & $0.692-2.300$ & 0.448 \\
\hline LVSI (positive vs. negative) & 1.247 & $0.749-2.076$ & 0.396 & 1.236 & $0.7 \mid I-2.148$ & 0.453 \\
\hline SM (positive vs. negative) & 3.249 & I.279-8.249 & 0.013 & 3.697 & $0.7 \mid 1-2.148$ & 0.007 \\
\hline PI (positive vs. negative) & 0.418 & $0.100-1.752$ & 0.233 & 0.251 & $0.034-1.84$ & 0.174 \\
\hline $\begin{array}{l}\text { Histologic type (adenocarcinoma and } \\
\text { adenosquamous carcinoma vs. SCC) } \\
\text { FIGO } 2018 \text { stage }\end{array}$ & 1.081 & $0.621-1.88 \mid$ & 0.784 & 1.036 & $0.562-1.910$ & 0.911 \\
\hline $\mid \mathrm{B} 2$ vs. IBI & 1.204 & $0.481-3.013$ & 0.692 & 1.180 & $0.4 \mid 4-3.367$ & 0.757 \\
\hline IB3 vs. IBI & 1.428 & $0.428-4.765$ & 0.562 & 1.704 & $0.454-6.398$ & 0.430 \\
\hline$\| A \mid$ vs. IBI & 1.293 & $0.494-3.382$ & 0.600 & 1.703 & $0.610-4.754$ & 0.310 \\
\hline$\| A 2$ vs. IBI & 1.763 & $0.556-5.588$ & 0.336 & 2.390 & $0.683-8.364$ & 0.173 \\
\hline IIICIP vs. IBI & 2.597 & $1.055-6.393$ & 0.038 & 3.246 & $1.202-8.763$ & 0.020 \\
\hline IIIC2P vs.IBI & 9.773 & $3.340-25.598$ & $<0.001$ & II.588 & $3.594-37.358$ & $<0.001$ \\
\hline
\end{tabular}

Abbreviations: OS, Overall survival; PFS, progression-free survival; DSI, depth of stromal invasion; LVSI, lymphovascular space invasion; SM, surgical margin; PI, parametrial invasion; SCC, squamous cell carcinoma; FIGO, International Federation of Gynecology and Obstetrics. 
number of metastatic lymph nodes. Therefore, we suggest that during the next revision of the staging system, the FIGO committee should take into account the influence of the number of lymph node metastases on survival and prognosis of IIIC1P patients.

We recognize several limitations in our study. First, this was a retrospective study and had all the inherent limitations of this form of research. Second, the sample size was small. Third, all patients were from a single center and so the results may be generalizable to all patients.

In conclusion, the current FIGO 2018 staging system for cervical cancer appears to be useful for predicting survival in patients with risk factors after radical surgery. However, the survival of patients with stage IIA1 was better than that of stage IB3. Stage IIIC1 cervical cancer is not homogenous; survival in stage IIIC1p varies with the number of metastatic lymph nodes. Efforts should be made to further improve the FIGO staging system.

\section{Abbreviation list}

CCRT, concurrent chemoradiotherapy; DSI, depth of stromal invasion; FIGO, International Federation of Gynecology and Obstetrics; LVSI, lymphovascular space invasion; OS, overall survival; PALN, para-aortic lymph node; PFS, progression-free survival; PI, parametrial invasion; PLN, pelvic lymph node; RT, radiotherapy; RTOG, Radiation Therapy Oncology Group.

\section{Acknowledgment}

This study was supported by the Zhejiang Province Health Department Foundation (No.2015KYB068).

\section{Disclosure}

The authors report no conflicts of interest in this work.

\section{References}

1. Sedlis A, Bundy BN, Rotman MZ, Lentz SS, Muderspach LI, Zaino RJ. A randomized trial of pelvic radiation therapy versus no further therapy in selected patients with stage IB carcinoma of the cervix after radical hysterectomy and pelvic lymphadenectomy: a Gynecologic Oncology Group Study. Gynecol Oncol. 1999;73 (2):177-183. doi:10.1006/gyno.1999.5387

2. Van de Putte G, Lie AK, Vach W, Baekelandt M, Kristensen GB. Risk grouping in stage IB squamous cell cervical carcinoma. Gynecol Oncol. 2005;99(1):106-112. doi:10.1016/j.ygyno.2005.05.026

3. Ryu SY, Park SI, Nam BH, et al. Is adjuvant chemoradiotherapy overtreatment in cervical cancer patients with intermediate risk factors? Int $J$ Radiat Oncol Biol Phys. 2011;79(3):794-799. doi:10.1016/j.ijrobp.2009.11.019
4. Samlal RA, van der Velden J, Schilthuis MS, et al. Identification of high-risk groups among node-positive patients with stage IB and IIA cervical carcinoma. Gynecol Oncol. 1997;64(3):463-467.

5. Inoue T, Okumura M. Prognostic significance of parametrial extension in patients with cervical carcinoma Stages IB, IIA, and IIB. A study of 628 cases treated by radical hysterectomy and lymphadenectomy with or without postoperative irradiation. Cancer. 1984;54 (8):1714-1719.

6. Quinn MA, Benedet JL, Odicino F, et al. Carcinoma of the Cervix Uteri. Int $J$ Gynaecol Obstet. 2006;95 Suppl 1:S43-S103. doi:10.1016/S0020-7292(06)60030-1

7. Bhatla N, Aoki D, Sharma DN, Sankaranarayanan R. Cancer of the cervix uteri. Int $J$ Gynaecol Obstet. 2018;143 Suppl 2:22-36. doi:10.1002/ijgo.12611

8. Matsuo K, Machida H, Mandelbaum RS, Konishi I, Mikami M. Validation of the 2018 FIGO cervical cancer staging system. Gynecol Oncol. 2019;152(1):87-93. doi:10.1016/j. ygyno.2018.10.026

9. Odicino F, Pecorelli S, Zigliani L, Creasman WT. History of the FIGO cancer staging system. Int J Gynaecol Obstet. 2008;101 (2):205-210. doi:10.1016/j.ijgo.2007.11.004

10. Noguchi H, Shiozawa I, Sakai Y, Yamazaki T, Fukuta T. Pelvic lymph node metastasis of uterine cervical cancer. Gynecol Oncol. 1987;27(2):150-158.

11. Liu Y, Zhao LJ, Li MZ, Li MX, Wang JL, Wei LH. The number of positive pelvic lymph nodes and multiple groups of pelvic lymph node metastasis influence prognosis in stage IA-IIB cervical squamous cell carcinoma. Chin Med J. 2015;128(15):2084-2089. doi:10.4103/0366-6999.161372

12. Berman ML, Keys H, Creasman W, DiSaia P, Bundy B, Blessing J. Survival and patterns of recurrence in cervical cancer metastatic to periaortic lymph nodes (a Gynecologic Oncology Group study). Gynecol Oncol. 1984;19(1):8-16.

13. Matsuo K, Grubbs BH, Mikami M. Quality and quantity metrics of pelvic lymph node metastasis and risk of para-aortic lymph node metastasis in stage IB-IIB cervical cancer. J Gynecol Oncol. 2018;29(1):e10. doi:10.3802/jgo.2018.29.e10

14. Kim HS, Song YS. International Federation of Gynecology and Obstetrics (FIGO) staging system revised: what should be considered critically for gynecologic cancer? J Gynecol Oncol. 2009;20(3):135-136.

15. Brockbank E, Kokka F, Bryant A, Pomel C, Reynolds K. Pretreatment surgical para-aortic lymph node assessment in locally advanced cervical cancer. Cochrane Database Syst Rev. 2013;(3): CD008217.

16. Grigsby PW, Heydon K, Mutch DG, Kim RY, Eifel P. Long-term follow-up of RTOG 92-10: cervical cancer with positive para-aortic lymph nodes. Int J Radiat Oncol Biol Phys. 2001;51(4):982-987.

17. Gouy S, Morice P, Narducci F, et al. Prospective multicenter study evaluating the survival of patients with locally advanced cervical cancer undergoing laparoscopic para-aortic lymphadenectomy before chemoradiotherapy in the era of positron emission tomography imaging. J Clin Oncol. 2013;31(24):3026-3033. doi:10.1200/ JCO.2012.47.3520

18. Inoue T, Morita K. The prognostic significance of number of positive nodes in cervical carcinoma Stages IB, IIA, and IIB. Cancer. 1990;65 (9):1923-1927.

19. Sakuragi N, Satoh C, Takeda N, et al. Incidence and distribution pattern of pelvic and paraaortic lymph node metastasis in patients with Stages IB, IIA, and IIB cervical carcinoma treated with radical hysterectomy. Cancer. 1999;85(7):1547-1554.

20. Takeda N, Sakuragi N, Takeda M, et al. Multivariate analysis of histopathologic prognostic factors for invasive cervical cancer treated with radical hysterectomy and systematic retroperitoneal lymphadenectomy. Acta Obstet Gynecol Scand. 2002;81 (12):1144-1151. 


\section{Publish your work in this journal}

Cancer Management and Research is an international, peer-reviewed open access journal focusing on cancer research and the optimal use of preventative and integrated treatment interventions to achieve improved outcomes, enhanced survival and quality of life for the cancer patient.
The manuscript management system is completely online and includes a very quick and fair peer-review system, which is all easy to use. Visit http://www.dovepress.com/testimonials.php to read real quotes from published authors. 